\title{
Análisis de las Técnicas de Traducción y las Figuras Literarias aplicadas a la versión en español del cuento Le Petit Prince (El Principito)
}

\author{
Analysis of the Translation Techniques and Literary Figures applied to the spanish \\ version of Le Petite Prince (The Little Prince)
}

Sandra García Cruz*

Perla del Rocío Rojas León**

Jesús Eduardo Gordillo Fuentes***

\begin{abstract}
* Sandra García Cruz. Egresada de la Licenciatura en Idiomas DAEA-UJAT. Participó en Movilidad Estudiantil Nacional en la Universidad Autónoma de Querétaro y Movilidad Estudiantil Internacional en la Universidad de Sherbrooke, Canadá. Participó en el programa de Verano Científico en el CEPHCIS, UNAM en Yucatán.
\end{abstract}

** Perla del Rocío Rojas León. Licenciada en Idiomas. Maestra en Administración con especialidad en Dirección de Factor Humano. Doctora en Educación. Profesora de Tiempo Completo DAEA-UJAT

*** Eduardo Jesús Gordillo Fuentes. Licenciatura en Administración de Empresas, maestría en Psicología y Orientación Vocacional, Doctorado en Ciencias de la Educación, postdoctorado en Innovación y Calidad Educativa para el Desarrollo, Perfil deseable y Apoyo (PRODED), miembro del Sistema Estatal de Investigadores, Miembro del Colegio de Doctores en Educación en Tabasco, a.c.

\section{RESUMEN}

En el presente artículo se expone un resumen del análisis de la traducción al español realizada por el traductor mexicano José María Francés, del cuento escrito en francés Le Petit Prince, de Antoine de Saint-Exupéry (1943). Este análisis se centraliza en las técnicas de traducción que utiliza el traductor en la adaptación del francés al español y su aplicación en las figuras literarias que se encuentran dentro de su respectivo contexto. El análisis se basa en las propuestas de Vázquez de Ayora (1977).

Palabras clave: El Principito, figuras literarias, traducción literaria, técnicas de traducción.

\section{ABSTRACT}

This article presents a summary of the analysis of the Spanish translation carried out by the Mexican translator José Maria Francés, from the French story Le Petit Prince (The Little Prince), by Antoine de Saint-Exupéry. This analysis is centralized in the translation techniques used by the translator in the adaptation of French to Spanish and its application in literary figures, which are within their respective context. The analysis is based on Vazquez de Ayora's translation techniques (1977).

Key words: The Little Prince, figures of speech, literary translation, translation techniques.

\section{INTRODUCCIÓN}

El libro Le Petit Prince se trata de una obra de género narrativo publicada en 1943 por Antoine de Saint-Exupéry, que puede considerarse novela por su escritura en prosa, pero al mismo tiempo forma parte de la literatura infantil y puede considerársele como cuento también. Mayormente se le ha contemplado desde una perspectiva dirigida hacia los niños, aunque esto no debería ser así, pues se tratan temas tan profundos como el sentido de la vida, la amistad, el amor y reflexiones en cuestiones de la vida adulta. Por ello, los infantes que en su temprana edad aún no desarrollan un pensamiento contextualizado en la experiencia de la vida adulta, no pueden comprenderlo sino de manera literal, sin descifrar el sentido figurado de la lectura. En general, se trata de una metáfora que hay que deducir; asimismo está comprendido de otras pequeñas metáforas, que si no se desvelaran, sería muy difícil entender el significado de la obra entera. Un mismo enunciado podría ser interpretado de diferentes maneras por distintos lectores y cada opinión sería una verdad, desde su punto de vista, no habiendo una sola manera de interpretar las alusiones que hace Saint-Exupéry. Además, podemos encontrar muchas figuras literarias inmersas dentro del relato, las cuales le dan sentido y estructura a sus alegorías.

Esta investigación tiene como objetivo, realizar un análisis de esas figuras literarias presentes en el cuento original Le Petit Prince, así como las técnicas de traducción aplicadas en la versión al español por el traductor mexicano José M. Francés.

El estudio y explicación de las figuras literarias permitirá a todos aquellas personas que de niños dieron lectura a El Principito 
Perspectivas Docentes 64

Análisis de las Técnicas de Traducción y las Figuras Literarias aplicadas a la versión en español del cuento Le Petit Prince (El Principito)

Analysis of the Translation Techniques and Literary Figures applied to the spanish version of Le Petite Prince (The Little Prince)

Sandra García Cruz / Perla del Rocío Rojas León / Jesús Eduardo Gordillo Fuentes ${ }^{\star \star \star}$

y no habían comprendido su mensaje, descifrar parte de las insinuaciones que el autor nos expresa en su relato, y también despertará el interés de entenderlo desde otra perspectiva a quienes deseen leerlo en la edad adulta, ya sea por primera 0 reiteradas veces. Analizar las técnicas de traducción aplicadas a la versión en español, será útil para quienes desean adentrarse en la traducción literaria y consideren las técnicas de traducción propuestas por Vázquez-Ayora (1977), para efectuar las equivalencias necesarias desde la lengua origen a la lengua meta. Asimismo, que tengan a bien tomar esta investigación como herramienta conveniente en futuras traducciones.

Los objetivos de este trabajo de investigación fueron entre otros: Comparar las figuras literarias presentes en el cuento original del cuento Le Petit Prince con las de la versión traducida al español. Describir la forma en que el traductor representa dichas figuras en su versión. Analizar las técnicas de traducción empleadas por el traductor en la adaptación al español desde la versión original y definir cuáles son las técnicas de traducción y figuras literarias más concurrentes en la comparación de las dos versiones del cuento.

\section{LA TRADUCCIÓN Y EL TRADUCTOR LITERARIO}

Desde hace muchos años, la traducción ha sido uno de los procedimientos más significativos para la expansión de la cultura, para la creación y desarrollo de nuevas literaturas y para el enriquecimiento de los idiomas empleados para traducir. Más que una ciencia por su naturaleza técnica, puede considerarse un arte, ya que el traductor en el proceso no se limita a solamente reemplazar una palabra de una lengua extranjera por otra del idioma propio, sino que se trata más bien de recrear el texto original en la lengua meta y esto permite crear un vínculo entre las ideologías culturales de dos países. Además, se puede asegurar que la traducción debe ir de la mano con la cultura, porque si bien es indiscutible que hay cualidades en una lengua que la diferencia de otras, no siempre se acude al estudio de los idiomas en función con la cultura que representa. Este es un aspecto importante que el traductor no debe descuidar para que su traducción sea enteramente apegada a la versión original y pueda contrastar las dos culturas con las que está tratando. Respecto a lo anterior, parece importante enfatizar en las propuestas de Lucía Luque (2010: 24-17), quien apoya su relación entre lengua y cultura en la clasificación de las culturas propuestas por Hall (1976), Copeland y Griggs (1986), entre otros.
Octavio Paz, quien además de ser escritor también fungió como traductor, afirma que el traductor literario debe tener rasgos comunes con el escritor, porque su función es la de re-elaborar en la lengua meta un mensaje que debe ser equivalente al original tanto en materia de contenido, como de forma (1990:21). Del mismo modo, Ayala sostiene que el traductor literario debe "poseer las aptitudes y la formación de escritor. Estas aptitudes solo pueden ejercerse en la lengua propia", de la que se espera, tenga un buen dominio que le permita solucionar las numerosas dificultades que puedan presentarse en la traducción de una obra literaria, citado por García Yebra (1989: 133). Estas dificultades, por lo general, son de categoría estilística y cultural. Dichos rasgos se encuentran relacionados con la "competencia literaria", que está compuesta, según Hurtado Albir, por "amplios conocimientos literarios y culturales y determinadas aptitudes relacionadas con el funcionamiento de esos textos (buenas habilidades de escritura, creatividad, etc.)". Esta competencia le facilitará re-elaborar en el texto traducido, aspectos de estética que pueda encontrar en el texto original, tales como las figuras literarias, el estilo y las connotaciones.

Se podría mal interpretar que el traductor literario se encarga de "adornar" el texto lo más posible, sin embargo su labor se trata de utilizar diversas técnicas que le permitan expresarse lo mejor posible en su adaptación a la lengua meta pero sin perder el estilo original del autor, para ello también podría hacer uso de la literalidad cuando se requiera. Tanto Savoy (1957) como Reiss (1971) han afirmado que al traductor técnico le interesa el contenido y al literario la forma. Otros autores sostienen que una traducción técnica debe ser literal y que una traducción literaria ha de ser libre; otros afirman lo contrario.

Estaríamos de acuerdo con la afirmación que al traductor literario le interesa la forma, si bien debe concentrarse en las ideas originales del autor y plasmarlas en el idioma propio con la mayor precisión posible, también tiene la libertad de elegir entre diversos elementos que las técnicas de traducción le otorgan para enriquecer el texto, al igual que le permita emplear el vocabulario y el acervo que domine.

El traductor también debe adquirir competencias que le permitan hacer una buena adaptación del texto en la lengua meta, porque si bien es cierto que debe hacer una re-elaboración del texto original, siempre es necesario conservar las ideas originales, y como resultado se obtendrá un producto final satisfactorio para los lectores. Respecto a esto, Pierre Daniel Huet, nos dice que "la traducción mejor no es la que aumenta la lozanía del autor, o remedia su aridez, o aclara su oscuridad, 
Perspectivas Docentes 64

Análisis de las Técnicas de Traducción y las Figuras Literarias aplicadas a la versión en español del cuento Le Petit Prince (El Principito)

Analysis of the Translation Techniques and Literary Figures applied to the spanish version of Le Petite Prince (The Little Prince)

Sandra García Cruz / Perla del Rocío Rojas León / Jesús Eduardo Gordillo Fuentes***

o corrige sus errores o establece orden en su desorden, sino la que pone ante nuestros ojos al autor entero, iluminado con sus propios colores, y nos lo entrega para que lo alabemos por sus genuinas virtudes 0 , si así lo ha merecido, nos riamos de sus peculiares vicios", es por eso que el traductor debe hacer uso de su creatividad y sus competencias lingüísticas para lograr si es posible, una obra de arte en su texto traducido, citado por García Yebra (1989: 223)

Siguiendo en el aspecto competitivo del traductor, expertos como Scott Tennent et al. (2000) consideran que la competencia de sus estudiantes de traducción debe basarse, principalmente, en desarrollar una habilidad para reconocer y solucionar problemas específicos, tal como lo explican en el siguiente fragmento:

"Bearing in mind the pedagogical focus of this study, the main aim was that students should develop the ability to recognize and solve these translationsécific problems: problem recognition is a pre-requisite for problem-solving" (2000: 108)

Si bien el autor anterior se refiere aún a los estudiantes, es un punto importante a tener en cuenta para llegar a ser un profesional en materia de traducción.

\section{PROCEDIMIENTO GENERAL DE LA TRADUCCIÓN}

En la propuesta de Vázquez-Ayora (1977), se presenta el procedimiento general de la traducción. Este se basa principalmente en cuatro aspectos:

1. La lectura del texto: explica que "antes de cometer cualquier traducción se debe dar lectura atenta y completa al texto. Sería ilógico querer traducir un texto sin la observación de sus caracteres estilísticos. Es necesario determinar a grandes rasgos los hechos del discurso que se van a encarar". Es indispensable realizar una lectura previa al texto original y detectar los elementos importantes presentes desde el principio. Tal como debió hacerlo José M. Francés, con el cuento "Le Petit Prince", lo que le permitió identificar poco a poco las figuras literarias y posteriormente buscar sus equivalencias en el idioma español.
2. Acervo intelectual y cultural del lector: "el análisis será, en consecuencia el fruto no solo del simple conocimiento de las lenguas, sino de la capacidad de comprender el comportamiento de las estructuras y de manejarlas en la forma más productiva. En cuanto al tema concreto que deba abordar el traductor, su acervo estará determinado por sus estudios generales o especializados, sus lecturas y por las traducciones anteriores". En esta se refiere a la capacidad de comprensión que el traductor tenga, así como el amplio vocabulario que debe poseer para lograr reflejar al autor de la mejor manera en la lengua meta.

3. Documentación: "la constituyen las lecturas referidas a la preparación inmediata del tema, hay que informarse sobre la materia que se va a traducir. Para ello sirve la consulta de obras sobre el mismo tema, artículos, traducciones anteriores."

4. El proyecto de traducción: "después de las fases anteriores, el traductor procede a realizar la primera versión, aplicando los principios, procedimientos y técnicas bosquejadas en el presente estudio". Aquí es donde el traductor procede a aplicar las técnicas de traducción requeridas según el texto, para reestructurar las ideas en la lengua meta, ya que el problema de este radica precisamente en la enorme dificultad de entender el pasaje de un texto y dejar de un lado la lengua original para concentrarse exclusivamente en la propia.

El buen empleo de cada uno de estos elementos depende del traductor, pues una buena traducción va a lograrse si este posee una enorme riqueza intelectual, cultural, y documentación.

\section{LAS FIGURAS LITERARIAS Y LAS TÉCNICAS DE TRADUCCIÓN}

Las figuras literarias son algunas de las dificultades que se le presentan al traductor literario. Estas son formas no convencionales de emplear las palabras para dotarlas de expresividad, vivacidad o belleza, con el objeto de sorprender, emocionar sugerir o persuadir. Existe una gran variedad y generalmente los autores se caracterizan por la utilización de algunas de ellas. Si bien en el cuento Le Petit Prince se encuentran muchas, en este estudio solo se analizan algunas de las más frecuentes: 
Perspectivas Docentes 64

Análisis de las Técnicas de Traducción y las Figuras Literarias aplicadas a la versión en español del cuento Le Petit Prince (El Principito)

Analysis of the Translation Techniques and Literary Figures applied to the spanish version of Le Petite Prince (The Little Prince)

Sandra García Cruz / Perla del Rocío Rojas León / Jesús Eduardo Gordillo Fuentes ${ }^{\star \star *}$

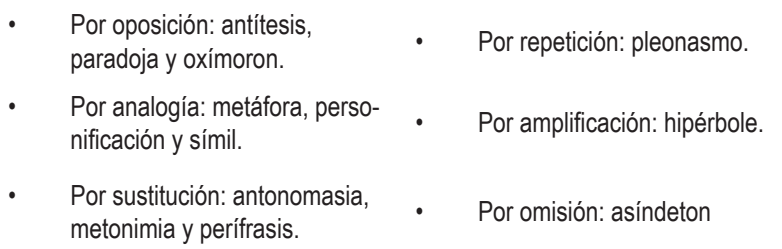

Las técnicas de traducción son la herramienta con la cual el traductor supera las dificultades de la traducción. Hurtado Albir (2001) las define como el "procedimiento visible en el resultado de la traducción, que se utiliza para conseguir la equivalencia traductora a micro unidades textuales". Esta autora presenta en Traducción y Traductología una recopilación de las técnicas de traducción más reconocidas por los expertos del campo de la traducción, sin embargo, se toma en cuenta la clasificación de Vázquez -Ayora, las cuales son traducción literal, transposición, modulación, equivalencia, adaptación, amplificación, explicitación, omisión y compensación.

\section{MATERIALES Y MÉTODOS}

La presente investigación es de enfoque cualitativo, ya que la herramienta con la que se llevó a cabo el estudio fue el libro Le Petit Prince del autor francés Antoine de Saint-Exupéry (1943), y con la versión en español traducida por el mexicano José M. Francés (1964). Para realizar el análisis, primeramente se leyó el libro original, que permitiría tener una idea general de la historia. Después se leyó la adaptación en español, y fue entonces que surgió la curiosidad por estudiar el proceso de traducción que se había efectuado. Finalmente se examinaron los dos textos de manera simultánea, haciendo lecturas más profundas y atendiendo minuciosamente los detalles de la traducción, tales como las técnicas de traducción utilizadas por el traductor y los cambios estructurales y estilísticos del texto.

Estas últimas lecturas se realizaron con base en dos aspectos: primeramente, en la búsqueda de las figuras literarias en el texto original y en el análisis de su traducción, lo cual nos permitió percibir cuándo estas figuras se eliminaban o permanecían. Luego, se procedió a identificar las técnicas de traducción utilizadas por José M. Francés, lo que nos permitiría revelar si utiliza las técnicas recopiladas por Vázquez de Ayora. Por ello, se le atribuye un alcance exploratorio a la investigación.

\section{ANÁLISIS DE LAS TÉCNICAS DE TRADUCCIÓN DE LE PETIT PRINCE.}

\section{TRADUCCIÓN LITERAL}

Técnica que designa el paso de la lengua de partida a la lengua de llegada que da lugar a un texto que es al mismo tiempo correcto e idiomático sin que el traductor haya tenido que preocuparse más que de las servidumbre idiomáticas:

[...] -Quand tu regarderas le ciel, la nuit, puisque j'habiterai dans l'une d'elles, puisque je rirai dans l'une d'elles, alors ce sera pour toi comme si riaient toutes les étoiles. Tu auras, toi, des étoiles qui savent rire! (p. 99)

[...] -Cuando mires al cielo por la noche, como viviré en una de ellas, como reiré en una de ellas, para ti será como si rieran todas las estrellas. ¡Tú tendrás estrellas que saben reír! (p. 110)

En los dos párrafos observamos que existe una correspondencia, salvo por ciertos ajustes que obedecen a la sintaxis particular de cada lengua; se han realizado las adiciones optativas de alors y toi en francés, o la adición también optativa de la preposición por en español. Pero es importante notar que estas alteraciones no afectan a la traducción literal.

\section{TRANSPOSICIÓN}

Técnica de traducción por la cual se reemplaza una parte del discurso del texto origen (TO) por otra diferente que en el texto meta (TM) lleve el principal contenido semántico de la primera. Es un cambio de categoría gramatical:

[...] J'entrevis aussitôt une lueur, dans le mystère de sa présence, et j'interrogeai brusquement: (p. 13)

[...] Vislumbré en seguida un destello en el misterio de su presencia, y de improviso pregunté: (p. 17)

Al comparar las dos versiones, se puede percibir que ocurrió un cambio de categoría gramatical: el adverbio brusquement del francés pasó a ser un sustantivo (de improviso) en español. Este tipo de variaciones son muy frecuentes cuando se 
Perspectivas Docentes 64

Análisis de las Técnicas de Traducción y las Figuras Literarias aplicadas a la versión en español del cuento Le Petit Prince (El Principito)

Analysis of the Translation Techniques and Literary Figures applied to the spanish version of Le Petite Prince (The Little Prince)

Sandra García Cruz / Perla del Rocío Rojas León / Jesús Eduardo Gordillo Fuentes***

pretende evitar la repetición del fonema "mente" en el idioma español, y esto no altera el mensaje original.

\section{MODULACIÓN}

Técnica de traducción que consiste en variar la forma del mensaje mediante un cambio semántico o de perspectiva:

[...] La planète suivante était habitée par un buveur. Cette visite fut très courte, mais elle plongea le petit prince dans une grande mélancolie: (p. 50)

[...] En el planeta siguiente vivía un bebedor. Esta visita fue muy corta, pero sumió al principito en una gran melancolía: (p. 56)

En este caso, es notable el empleo de la modulación, ya que se realiza un cambio de perspectiva al evitar la voz pasiva del francés sobre la versión en español, dado que es menos frecuente utilizarla.

\section{ADAPTACIÓN}

Técnica de traducción en la que el traductor expresa un mismo mensaje con otra situación equivalente:

$$
\begin{aligned}
& \text { [...] - S'il vous plaît... dessine-moi un mouton! } \\
& \text { - } \text { Hein! } \\
& \text { - Dessine-moi un mouton... (p. 8) } \\
& \text { [...] - Por favor... dibújame un cordero. } \\
& \text { - ¿Qué? } \\
& \text { - Dibújame un cordero... (p. 10) }
\end{aligned}
$$

En este caso la onomatopeya Hein! del francés, no existe en español. Por consiguiente se tuvo que utilizar otra palabra (¿Qué?) de modo que esta reemplazara a la original, causando el mismo efecto en la lengua española.

\section{AMPLIFICACIÓN}

Técnica de traducción que consiste en una expansión del sentido de una categoría gramatical, principalmente de una preposición, de un TO a un TM para expresar la misma idea:
[...] J'ai ainsi vécu seul, sans personne avec qui parler véritablement, jusqu'à une panne dans le désert du Sahara, il y a six ans. (p. 8)

[...] Así, pues, viví solo, sin tener con quien hablar de verdad, hasta que tuve una avería en el desierto del Sahara hace seis años. (p.10)

Aquí es evidente que se ha realizado una amplificación de la preposición à en francés. En esta lengua se entiende perfectamente el mensaje tal cual está escrito, sin embargo, en español fue necesario extenderla un poco más para que el enunciado adquiriera coherencia.

\section{OMISIÓN}

Consiste en eliminar elementos redundantes para obtener una traducción más concisa por razones estructurales o estilísticas:

[...]-Mais les graines sont invisibles. Elles dorment dans le secret de la terre jusqu'à ce qu'il prenne fantaisie à l'une d'elles de se réveiller. Alors elle s'étire, et pousse d'abord timidement vers le soleil une ravissante petite brindille inoffensive. (p. 22)

[...] -Pero las semillas son invisibles. Duermen en el secreto de la tierra hasta que a una de ellas se le antoja despertarse. Entonces, se estira y, tímidamente al principio, crece hacia el sol una encantadora ramita inofensiva. (p. 26, 27)

Al comparar los dos párrafos, se observa que el traductor hizo la omisión del artículo elle en francés. No es necesario repetirlo en español, ya que se sobreentiende que se habla del mismo objeto en las oraciones subsecuentes y descartarlo no genera ningún cambio en su significado. Sin embargo, repetirlo, alteraría la fonética natural del idioma. 
Perspectivas Docentes 64

Análisis de las Técnicas de Traducción y las Figuras Literarias aplicadas a la versión en español del cuento Le Petit Prince (El Principito)

Analysis of the Translation Techniques and Literary Figures applied to the spanish version of Le Petite Prince (The Little Prince)

Sandra Garcia Cruz / Perla del Rocío Rojas León / Jesús Eduardo Gordillo Fuentes ${ }^{\star \star *}$

\section{FIGURAS LITERARIAS PRESENTES EN LA OBRA ORIGINAL EN FRANCÉS}

\section{A) ANÁFORA}

Consiste en la reiteración de una o más palabras al comienzo de una frase o verso, o al inicio de varias frases o versos integrantes de un período o de una estrofa o poema:

[...] -Je connais une planète où il y a un Monsieur cramoisi. $\|$ n'a jamais respiré une fleur. $\|$ n'a jamais regardé une étoile. $\|$ n'a jamais aimé personne. $\|$ n'a jamais rien fait d'autre que des additions. (p. 30)

[...] -Conozco un planeta en el que hay un señor coloradote. Nunca ha olido una flor. Nunca ha mirado una estrella. Nunca ha querido a nadie. Nunca ha hecho más que sumas. (p. 34)

En este párrafo, el escritor describe la actitud que un cierto personaje tiene hacia la vida. Aparentemente desea enfatizar las acciones que jamás ha realizado y atraer la atención del lector hacia ello; es por eso que la frase se vuelve repetitiva. José M. Francés realiza una traducción literal de la figura literaria para lograr el mismo efecto en español.

\section{B) ANTÍTESIS}

Es una contraposición de dos palabras o frases de significación opuesta, que adquieren así mayor expresividad y viveza. Este contraste ocurre, a veces, oponiendo dos palabras antónimas o frases enteras:

[...]-Je me demande, dit-il, si les étoiles sont éclairées afin que chacun puisse un jour retrouver la sienne. Regarde ma planète. Elle est juste au-dessus de nous... Mais comme elle est loin! (p. 68)

[...] -Me pregunto -dijo- si las estrellas no estarán iluminadas para que cada uno pueda un día encontrar la suya. Mira mi planeta. Está exactamente encima de nosotros... Pero iqué lejos está! (p.78)
En este caso, el Principito conversa con el escritor (que es a la vez personaje de su propio cuento), expresándole un poco su melancolía por la distancia que se interpone entre ciertas entidades. La utilización de la antítesis coincide con el sentimiento del Principito, quien a pesar de percibir tan de cerca su planeta, en realidad se encontraba muy lejos de él. La figura refleja muy bien la presencia de términos contradictorios dentro de una sola oración (lejanía/cercanía). La traducción de esta figura es en su totalidad literal.

\section{C) HIPÉRBOLE}

Consiste en ofrecer una visión desproporcionada de una realidad, amplificándola o disminuyéndola:

[...] -Ta planète est tellement petite que tu en fais le tour en trois enjambées. Tu n'as qu'à marcher assez lentement pour rester toujours au soleil. (p. 60)

[...]-Tu planeta es tan pequeño, que, de tres zancadas, le puedes dar la vuelta. No tienes más que andar bastante despacio para estar siempre al sol. (p. 67)

En este ejemplo de hipérbole, Saint-Exupéry pretende proyectar las extremadamente pequeñas dimensiones de otro planeta donde se hallaba el Principito, insinuando que no era preciso hacer gran esfuerzo para recorrerlo por completo. Vemos que en este caso, solo se hizo un pequeño cambio en el orden del enunciado en español, pero por la similitud de las dos lenguas, la figura se tradujo de manera literal sin ninguna modificación en el significado.

\section{D) METÁFORA}

[...] Ma vie est monotone. Je chasse les poules, les hommes me chassent. Toutes les poules se ressemblent, et tous les hommes se ressemblent. Je m'ennuie donc un peu. Mais, si tu m'apprivoises, ma vie sera comme ensoleillée. Je connaîtrai un bruit de pas qui sera différent de tous les autres. (p. 80)

[...] Mi vida es monótona. Yo cazo gallinas, los hombres me cazan a mí. Todas las gallinas se parecen y todos los hombres se parecen. Por eso me aburro un poco. Pero, si me domesticas será como si mi vida se bañara 
Perspectivas Docentes 64

Análisis de las Técnicas de Traducción y las Figuras Literarias aplicadas a la versión en español del cuento Le Petit Prince (El Principito)

Analysis of the Translation Techniques and Literary Figures applied to the spanish version of Le Petite Prince (The Little Prince)

Sandra García Cruz / Perla del Rocío Rojas León / Jesús Eduardo Gordillo Fuentes***

de sol. Conoceré un ruido de pasos que será diferente de los otros. (p. 88)

La metáfora de este fragmento se centra en la actitud del zorro, quien bajo una condición propuesta con anterioridad, pretende concebir una percepción positiva del Principito en comparación con todos los demás individuos. Al expresar ma vie será comme ensoleillée, hace alusión al cambio radical que significaría el Principito en la vida del zorro después de domesticarlo, pues le llenaría la vida de dicha y felicidad. En español, José M. Francés traduce esta figura literaria como mi vida se bañará de sol. En este caso recurrió a la técnica de amplificación, que es notable al cabo de la frase, y esto con el fin de embellecerla en español sin distorsionar la idea original.

\section{E) PERSONIFICACIÓN}

Se atribuyen cualidades 0 acciones propias de seres humanos a animales, objetos o ideas abstractas:

[...] -mais la fleur n'en finissait pas de se préparer à être belle, à l'abri de sa chambre verte. Elle choisissait avec soin ses couleurs. Elle s'habillait lentement, elle ajustait un à un ses pétales. (p. 32)

[...] -pero la flor, al abrigo de su aposento verde, no acababa de arreglarse para estar guapa. Escogía con cuidado sus colores. Se vestía lentamente, ajustaba sus pétalos uno a uno. (p. 37)

En este fragmento completo hay un claro ejemplo de personificación, pues a la flor que es un objeto inanimado, se le atribuyen las acciones de una persona, especialmente las de una mujer; desde vestirse, arreglarse y hacer la elección de lo que va mejor con ella. El traductor emplea la omisión como técnica de traducción pues reduce la utilización del artículo elle en la figura literaria al español, pero conserva la idea original del autor.

\section{F) SÍMIL}

Consiste en relacionar dos términos entre sí por la semejanza 0 analogía que presentan las realidades designadas por ellos:
[...] -Les éruptions volcaniques sont comme des feux de cheminée. Évidemment sur notre terre nous sommes beaucoup trop petits pour ramoner nos volcans. (p. 38)

[...] - Las erupciones volcánicas se parecen a una chimenea cuando se prende. Evidentemente, en nuestra tierra somos demasiado pequeños para deshollinar nuestros volcanes. (p. 43)

Este ejemplo contiene la frase comme en francés, y en español se parece a, enlaces concurrentes para la formación de símiles. Se compara a los volcanes con las chimeneas, en relación con la forma en que los dos expulsan el humo desde sus orificios. Para la traducción de la figura, evidentemente se empleó la técnica de amplificación.

\section{RESULTADOS}

Dentro del listado de las figuras retóricas propuestas para analizar, durante la investigación solamente se presentaron las más concurrentes en el texto, además que no todas fueron utilizadas por el traductor.

Cabe señalar que las figuras más comunes en el cuento son la personificación y el símil, ya que la historia se desarrolla por completo como una metáfora, pues la mayoría de sus personajes son ficticios 0 inanimados a quienes se les atribuye una condición y/o acción humana, lo que daría lugar a la personificación. En otros casos se les compara con algún otro objeto 0 ente que se asemeja en alguna cualidad para dar cabida a la figura símil. Seguidamente se encuentra la metáfora, la hipérbole, la antítesis y por último la anáfora.

En lo que concierne a la traducción de la personificación, que es la figura literaria más recurrente, se puede afirmar que se conserva casi intacta en la lengua meta. José M. Francés lo traduce de manera parcialmente literal, porque aplica la técnica de omisión de los artículos (ella) que no son necesarios en español.

En cuanto a la aplicación de las técnicas de traducción, José M. Francés en su labor de traductor, consiguió ser lo más apegado posible a la obra original, conservando el sentido y empleando las equivalencias adecuadas, encargándose de que las figuras literarias originales no se distorsionaran y el relato conservara su esencia en la adaptación a la lengua española. Utilizó 
Perspectivas Docentes 64

Análisis de las Técnicas de Traducción y las Figuras Literarias aplicadas a la versión en español del cuento Le Petit Prince (El Principito)

Analysis of the Translation Techniques and Literary Figures applied to the spanish version of Le Petite Prince (The Little Prince)

Sandra García Cruz / Perla del Rocío Rojas León / Jesús Eduardo Gordillo Fuentes ${ }^{\star \star *}$

solamente algunas de las técnicas propuestas por Vázquez de Ayora (1977), y la que predominó fue la traducción literal.

\section{DISCUSIÓN}

Es preciso tener en cuenta que existen autores como Hurtado Albir, Newman, Vinay y Darbelnet, entre otros, que clasifican las técnicas de traducción de acuerdo con su propio criterio. Sin embargo, el motivo por el cual la investigación se basó en las técnicas de Vázquez de Ayora es que su enfoque concierne en la traducción literaria, y también da ejemplos en cuanto al proceso de la traducción literal.

En la traducción de obras literarias como El Principito, estas técnicas resultaron útiles y permitieron al traductor facilitar su labor. Como se pudo señalar anteriormente, José M. Francés empleó técnicas propuestas por Vázquez de Ayora, tales como modulación, transposición, traducción literal, adaptación, amplificación, entre otras.

Se asumía que el traductor haría diversas variaciones en la traducción de las figuras literarias, que utilizaría equivalencias más complejas para dar el mismo significado en español. Sin embargo, a pesar del estilo poético que maneja Saint-Exupéry en su escritura, emplea un lenguaje sencillo y comprensible, apto para cualquier lector. Por esta razón, el traductor no se ve forzado a traducirlas en un lenguaje rebuscado, dándole mayor lugar a la traducción literal en la obra.

\section{CONCLUSIONES}

La elaboración de este análisis nos permitió destacar las figuras utilizadas por Saint-Exupéry en Le Petit Prince, la manera como son traducidas y las técnicas de traducción aplicadas por José M. Francés. Se puede notar también que hay numerosas figuras literarias presentes en la obra, además que cada una tiene una interpretación similar cuando se comparan de manera paralela en las dos diferentes lenguas.

Se concluye con que José M. Francés hizo una traducción en su mayoría literal, porque la lengua francesa dispone de mucha similitud en léxico y gramática con el español. Por lo tanto, es menos complicado conservar el sentido y plasmarlo de manera casi intacta ante la presencia de figuras literarias. También la simplicidad de lenguaje con que fue escrita la obra original facilita este proceso.

Los traductores hoy en día, tienen la oportunidad de elegir entre las diversas técnicas propuestas por los expertos de la Traductología, y aun cuando tienen la libertad de emplear las que mejor se ajusten al estilo del texto, lo más sustancial será siempre reflejar el mensaje original del autor lo más intacto posible y que el lector ni siquiera perciba que el texto se trata de una traducción. Para ello es importante que desenvuelva su creatividad, y desarrolle sus competencias lingüísticas y culturales, tal como lo hace el José M. Francés en su traducción del cuento El Principito. 
Perspectivas Docentes 64

Análisis de las Técnicas de Traducción y las Figuras Literarias aplicadas a la versión en español del cuento Le Petit Prince (El Principito)

Analysis of the Translation Techniques and Literary Figures applied to the spanish version of Le Petite Prince (The Little Prince)

Sandra García Cruz / Perla del Rocío Rojas León / Jesús Eduardo Gordillo Fuentes ${ }^{* * *}$

\section{REFERENCIAS BIBLIOGRÁFICAS}

Albir, A. H. (marzo de 2016). Traducción Literaria: Técnicas y Procedimientos de la Traducción. Recuperado el 25 de enero de 2018, de https://www.asjp.cerist.dz/en/ downArticle/33/16/32/1763

Alonso, A. L. (2001). Aspectos culturales y traducción. La tradición literaria. Recuperado el 24 de enero de 2018, de https://www.uv.es/dpujante/PDF/CAP3/B/A_Luna_Alonso.pdf

Ávila, C. G., \& Carrillo, L. J. (11 de julio de 2014). Octavio Paz: traducción y relaciones literarias. Recuperado el 25 de enero de 2018, de http://web.uaemex.mx/plin/colmena/Colmena_83/ docs/traduccion_Paz.pdf

Ayora, G. V. (1977). Introducción a la Traductología. Recuperado el 24 de enero de 2018, de https://www.fiuxy. bz/threads/introduccion-a-la-traductologia-curso-basico-detraduccion-gerardo-vazquez-ayora-pdf.4811096/

Bardají, A. G. (2008). Procedimientos, técnicas, estrategias: operadores del proceso traductor . Recuperado el 24 de enero de 2018, de http://www.recercat.cat/bitstream/ handle/2072/8998/TREBALL\%20DE\%20RECERCA\%20 ANNA\%20GIL.pdf?sequence=1

Cavero, M. E. (1996). Literatura y traducción: problemas que plantea y situación actual. Recuperado el 24 de enero de 2018, de https://dialnet.unirioja.es/servlet/articulo?codigo=3204048

De Saint-Exupéry, A. (s.f.). El Principito. (J. M. Francés, Trad.) Mérida, Yucatán, México: Dante.

De Saint-Exupéry, A. (1943). Le Petit Prince. Recuperado de: http://www.cmls.polytechnique.fr/perso/tringali/documents/ st_exupery_le_pe tit_prince.pdf
Newmark, P. (1991). La teoría y el arte de la traducción. Recuperado el 20 de enero de 2018, de https://dialnet.unirioja. es/descarga/articulo/5476322.pdf

Pym, A. (2016). Teorías contemporáneas de la traducción. Recuperado el 25 de enero de 2018, de https://www.researchgate.net/profile/Anthony_Pym2/ publication/266557109_Teorias_contemporaneas_de_la_ traduccion/links/5750919908ae1c34b39c296b/Teoriascontemporaneas-de-la-traduccion.pdf

Ramírez, M. V., \& López, A. B. (11 de octubre de 2012). Análisis de estrategias y procedimientos traductológicos utilizados por el país en la traducción de títulos de prensa del español al inglés. Recuperado el 26 de enero de 2018, de http://revistaseug.ugr. es/index.php/sendebar/article/viewFile/35/35

Sánchez, I. P. (s.f.). Obtenido de http://www.siff.us.es/fil/ publicaciones/apuntes/ipliego/AA $\% 20$ Ejemplos $\% 20$ de $\% 20$ procedimientos $\% 20$ tecnicos.pdf

Vega, M., \& Pulido, M. (5 de enero de 2013). La historia de la traducción y de la teoría de la traducción en el contexto de los estudios de la traducción. Recuperado el enero de 24 de 2018, de http://www.redalyc.org/articulo.oa?id=265129587001

Yebra, V. G. (1994). Scribd. Recuperado el 24 de enero de 2018, de https://es.scribd.com/doc/254934801/ValentinGarcia-Yebra-Traduccion-Historia-y-Teoria-PDF 